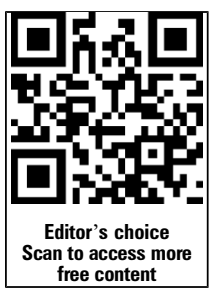

${ }^{1}$ Centre for Paediatrics and Child Health, Institute of Human Development, University of Manchester, Manchester, UK ${ }^{2}$ Department of Paediatric Endocrinology, Royal Manchester Children's Hospital, Central Manchester Foundation Hospitals NHS

Trust, Manchester Academic Health Science Centre, Manchester, UK

${ }^{3}$ Section of Genetics and Epigenetics in Health and Disease, Genetics and Genomic Medicine Programme, UCL Institute of Child Health, London, UK

${ }^{4}$ London Centre for Paediatric Endocrinology and Diabetes, Great Ormond Street Hospital For Children NHS Foundation Trust, London, UK

\section{Correspondence to} Dr Philip Murray, 5th Floor Research, Royal Manchester Children's Hospital, Oxford Road, Manchester M13 9WL, UK; Philip.Murray@manchester.ac.uk

Received 6 March 2015 Revised 12 June 2015 Accepted 15 June 2015 Published Online First

7 July 2015

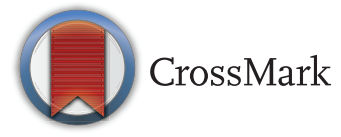

To cite: Murray PG, Dattani MT, Clayton PE. Arch Dis Child

2016;101:96-100.

\title{
Controversies in the diagnosis and management of growth hormone deficiency in childhood and adolescence
}

\author{
P G Murray, ${ }^{1,2}$ M T Dattani, ${ }^{3,4}$ P E Clayton ${ }^{1,2}$
}

\section{ABSTRACT}

Growth hormone deficiency (GHD) is a rare but important cause of short stature in childhood with a prevalence of 1 in 4000 . The diagnosis is currently based on an assessment of auxology along with supporting evidence from biochemical and neuroradiological studies. There are significant controversies in the diagnosis and management of GHD. Growth hormone (GH) stimulation tests continue to play a key role in GHD diagnosis but the measured $\mathrm{GH}$ concentration can vary significantly with stimulation test and GH assay used, creating difficulties for diagnostic accuracy. Such issues along with the use of adjunct biochemical markers such as IGF-I and IGFBP-3 for the diagnosis of GHD, will be discussed in this review. Additionally, the treatment of GHD remains a source of much debate; there is no consensus on the best mechanism for determining the starting dose of $\mathrm{GH}$ in patients with GHD. Weight and prediction based models will be discussed along with different mechanisms for dose adjustment during treatment (auxology or IGF-I targeting approaches). At the end of growth and childhood treatment, many subjects diagnosed with isolated GHD re-test normal. It is not clear if this represents a form of transient GHD or a false positive diagnosis during childhood. Given the difficulties inherent in the diagnosis of GHD, an early reassessment of the diagnosis in those who respond poorly to $\mathrm{GH}$ is to be recommended.

\section{INTRODUCTION}

Growth hormone deficiency (GHD) is a rare disorder with a prevalence of approximately 1 in 4000 during childhood. ${ }^{1}$ Although rare, it is an important diagnosis to make correctly-therapy with growth hormone (GH) in GHD is highly efficacious so a missed diagnosis will result in a poor outcome. Equally, a false positive diagnosis will lead to many years of daily subcutaneous injections, significant wasted expenditure ( $£ 7500$ per year) and unnecessary exposure to potential adverse effects. The diagnosis is multifaceted and includes an assessment of the patient's auxology, a biochemical assessment of the GH-IGF-I axis, and imaging of the hypothalamo-pituitary axis. Consensus guidelines on the diagnosis of GH deficiency in childhood were published in 2000 by the GH Research Society $^{2}$ (summary in box 1) and National Institute for Health and Care Excellence guidelines on the use of GH treatment were updated in $2010 .^{1}$

GH secretion exists in a continuum from normality through to severe GH deficiency. The diagnosis of GH deficiency is often very clear in a child with multiple pituitary hormone deficiencies (MPHD) or where the child presents with severe GH deficiency (typically with peak $\mathrm{GH}<3 \mu \mathrm{g} / \mathrm{L}$ ). Separating the group of short children with mild GH deficiency from non-GH deficient short children remains challenging. In this article we will review the data on diagnosis of GHD-which pharmacological stimulation test to use, issues with GH and IGF-I assays, requirement for priming and the effects of obesity. In addition we will also discuss how to select and adjust GH dose and identify non-responders to $\mathrm{GH}$ therapy.

\section{DIAGNOSIS OF GHD}

Outside the neonatal period (when a random GH measurement of $<7 \mu \mathrm{g} / \mathrm{L}$ identifies GHD in neonates), measurement of random serum GH concentrations are of no clinical value as $\mathrm{GH}$ secretion is pulsatile with the majority of GH pulses occurring overnight, with very low GH concentrations between pulses. This means that provocative tests of $\mathrm{GH}$ secretion using physiological/pharmacological stimuli are required to test for GHD. GH stimulation tests use a defined cut-off concentration for peak GH to distinguish GHD from non-GHD subjects. The lack of any 'gold standard' test for GHD diagnosis has led to the development of somewhat arbitrary cut-off levels. Attempts have been made to optimise the cut-off concentration using auxological criteria to define GHD (predominantly height velocity) but these attempts have been hampered as other disorders can share similar auxology to GHD. When GH stimulation tests were first used in the 1960 s, a peak GH concentration after stimulation of $<5 \mu \mathrm{g} / \mathrm{L}$ was used to diagnose GHD on the basis that this concentration seemed to best identify patients with a GHD phenotype. ${ }^{3}$ Over time, this cut-off has increased on the basis of very limited evidence to $7 \mu \mathrm{g} / \mathrm{L}$ and then $10 \mu \mathrm{g} / \mathrm{L}$ although most UK centres use a cut-off of $7 \mu \mathrm{g} / \mathrm{L}$ (A Chesover \& M Dattani, unpublished data). Recent studies by Wagner et al (discussed below) have suggested a peak GH cut-off of $7 \mu \mathrm{g} / \mathrm{L},{ }^{4}$ which is in line with the concentration generally used across the UK.

\section{Variability and reproducibility in peak $\mathrm{GH}$ concentration with different physiological or pharmacological stimuli}

The first test of GH secretion used was the insulin tolerance test. Subsequently a number of other pharmacological stimuli were identified including arginine, glucagon, clonidine, pyridostigmine, levodopa, GH releasing hormone (GHRH) and GHRH 
combined with arginine. Physical stimuli of GH secretion included sleep and exercise, but these have largely been abandoned as tests of GH secretion because of a lack of reproducibility. Most of the pharmacological tests are associated with side effects such as nausea, hypotension and somnolence. Very rarely, the insulin tolerance test has been associated with death due to hypoglycaemia or its overtreatment with high concentrations of dextrose, as has the glucagon test. ${ }^{5}$ In the UK the glucagon test and arginine stimulation test remain the most commonly used in clinical practice.

Ghigo et $a l^{6}$ have conducted the most comprehensive study to date comparing 10 different $\mathrm{GH}$ stimulation tests in 472 children without GHD. Mean peak GH concentrations varied between tests from $9.7 \mu \mathrm{g} / \mathrm{L}$ to $61.8 \mu \mathrm{g} / \mathrm{L}$. Excluding combined stimulation tests, all the tests incorrectly classified some subjects as GH deficient. Using a cut-off of $7 \mu \mathrm{g} / \mathrm{L}$, false positive rates varied between $8.9 \%$ and $23.7 \%$, depending on the test used, and increased to $14.9-49 \%$ when the cut-off was increased to $10 \mu \mathrm{g} / \mathrm{L}$. Variability in peak GH concentration also occurs between tests when used to investigate children with short stature. ${ }^{7}$ In addition to variability between the $\mathrm{GH}$ stimulation tests used, the reproducibility of stimulation tests is poor. ${ }^{8}$

Current UK guidelines recommend the use of two pharmacological tests for the diagnosis of idiopathic isolated GHD (see box 1). This strategy is used to improve diagnostic accuracy given the large number of false positive diagnoses from single stimulation tests in normal children.

Box 1 Consensus guidelines on the diagnosis and treatment of growth hormone (GH) deficiency in childhood and adolescence (GH Research Society ${ }^{2}$ )

\section{When to consider investigation for GH deficiency}

1. Severe short stature, defined as a height more than 3 SD below the mean.

2. Height more than $1.5 \mathrm{SD}$ below the mid-parental height.

3. Height more than 2 SD below the mean and a height velocity over 1 year more than 1 SD below the mean for chronological age, or a decrease in height SD of more than 0.5 over 1 year in children over 2 years of age.

4. In the absence of short stature, a height velocity more than 2 SD below the mean over 1 year or more than $1.5 \mathrm{SD}$ sustained over 2 years; this may occur in growth hormone deficiency (GHD), presenting in infancy, or in organic acquired GHD.

5. Signs indicative of an intracranial lesion.

6. Signs of MPHD.

7. Neonatal symptoms and signs of GHD.

\section{Investigations}

1. Once hypothyroidism has been excluded testing should proceed with a GH stimulation test and measurement of IGF-I I IGFBP-3 concentrations.

2. Pituitary MRI is required in all confirmed patients with $\mathrm{GH}$ deficiency in addition to those with known or suspected intracranial tumours, optic nerve hypoplasia/septo-optic dysplasia or other neurodevelopmental anomalies.

3. For isolated GHD two independent provocation tests are required. In those with a history of defined central nervous system pathology, history of irradiation, genetic defect known to cause GHD or multiple pituitary hormone deficiency, one GH test will suffice.
GH neurosecretory dysfunction and measurement of spontaneous GH secretion

As an alternative to GH stimulation tests, some groups began to assess physiological GH secretion using 12 -h or 24 -h profiles. This test requires hospital admission overnight and requires blood sampling every $20 \mathrm{~min}$. The reproducibility of $24-\mathrm{h} \mathrm{GH}$ profiles is superior to that of $\mathrm{GH}$ stimulation tests but it differentiates poorly between GH deficient and normal short children and has a lower sensitivity, failing to identify $57 \%$ of children classified as GHD via stimulation testing. ${ }^{9}$

GH neurosecretory dysfunction is defined as an abnormal 24-h GH secretory profile (reduced number of GH pulses and reduced pulse amplitude) in the presence of a low IGF-I concentration, auxology compatible with a diagnosis of GHD, bone age delay of at least 2 years and a normal GH stimulation test. A diagnosis of neurosecretory dysfunction should only be considered where the clinical presentation is strongly consistent with GHD, including structural abnormalities of the pituitary gland on MRI, but the pharmacological stimulation test is normal.

\section{Interassay variability and GH assay standardisation}

Technical as opposed to biological variability in peak GH is created through the use of different assay techniques. The UK National External Quality Assessment Scheme assesses variability across 96 clinical laboratories; in 1998 they reported a worsening overall variability in $\mathrm{GH}$ measurement over time with a geometric coefficient of variation of $25 \%$. This would result in a sample of GH with a mean measured concentration of $7 \mu \mathrm{g} / \mathrm{L}$ being reported by different laboratories with a range between 5 $\mu \mathrm{g} / \mathrm{L}$ and $10 \mu \mathrm{g} / \mathrm{L} .{ }^{10}$ Similar results have been reported in Germany, Finland and Japan. ${ }^{11}$

This interassay variability is thought to be the result of the molecular heterogeneity of $\mathrm{GH}$, variability in reference preparation used and effects of GH binding protein (GHBP). Each immunoassay will differ in the relative detection of the multiple GH variants present in serum due to alternative splicing, polymerisation and complexing with other molecules. ${ }^{12}$ The first two reference preparations used to calibrate $\mathrm{GH}$ assays used pituitary derived GH while standards produced from recombinant human $\mathrm{GH}$ were later introduced-each reference preparation contained different potencies of GH. Up to $50 \%$ of GH is bound in serum to GHBP and modern assays using monoclonal antibodies may be affected when the epitope of the antibody is obscured by the binding of GH to GHBP. Variations in GHBP within the physiological range have been found to significantly affect the GH concentration detected. ${ }^{13}$ The net effect of this assay variability can lead to significant differences in the diagnosis of GH deficiency between laboratories with the proportion classified as normal varying from $21 \%$ to $57 \%$ depending upon the assay used. ${ }^{14}$

Measurement of GH by mass spectrometry (MS) has the potential to circumvent many of the problems associated with immunoassays as it allows recognition by analyte mass rather than epitope. MS measurement of GH has been demonstrated to be completely independent of the serum concentration of $\mathrm{GHBP}^{15}$ and, unlike immunoassays, has the potential to establish a reproducible and sustainable cut-off concentration.

Wagner $e t a l^{4}$ recently re-examined the cut-off concentrations for the diagnosis of GHD for six currently available commercial immunoassays and for a MS based assay in 52 short children (without GHD) and 44 children diagnosed with GHD and treated with GH. Importantly, height standard deviation score (SDS) at stimulation test and height SDS at follow-up were comparable in both groups indicating that the classification of 
children into the non-GHD group was highly likely to be accurate. Serum samples from each child were re-examined on the iSYS assay and an optimal cut-off limit of $7.09 \mu \mathrm{g} / \mathrm{L}$ was identified and converted via regression equations to cut-off values for five other commercially available assays. An MS based assay was assessed in a subset of 30 patients and a cut-off concentration was derived from the iSYS cut-off by regression analysis. Table 1 lists the diagnostic cut-off values for each of the commercial assays and for the MS based assay.

\section{Priming for $\mathrm{GH}$ stimulation tests}

During puberty the activation of the hypothalamo-pituitary gonadal axis leads to a large increase in the circulating concentration of sex steroids that augments the pulse amplitude of $\mathrm{GH}$ secretion, and increases IGF-I concentrations and anterior pituitary size. Children in the peripubertal period and those with delayed puberty often display a decrease in growth velocity and short stature prompting an endocrine assessment. GH testing in this group frequently yields subnormal results but follow-up has indicated that many re-test with normal GH secretion upon reassessment. ${ }^{16}$ These data led to the suggestion that the primary pathology in these patients was sex steroid deficiency, with a probable diagnosis of constitutional delay. The use of oestrogen or testosterone to prime the $\mathrm{GH}$ axis prior to pharmacological stimulation tests has been demonstrated to increase peak $\mathrm{GH}$ concentrations and reduce false positive rates in healthy prepubertal subjects from $39 \%$ to $5 \% .{ }^{17}$ There is evidence from one follow-up study of 50 patients that not treating children with subnormal unprimed $\mathrm{GH}$ stimulation tests but normal primed $\mathrm{GH}$ stimulation tests does not result in an impaired final height. ${ }^{18}$

Within paediatric endocrinology there are two opposing schools of thought about priming: the first is that priming reduces the false positive rate of $\mathrm{GH}$ stimulation tests in peripubertal patients and should be used. The second and opposite view is that priming only briefly augments the $\mathrm{GH}$ response which then returns to suboptimal concentrations and may result in failure to treat children with transient peripubertal GH deficiency. As a result there are three strategies applied to priming by paediatric endocrinologists:

1. No priming

2. Sex steroid priming for children with pubertal delay (prepubertal at 13-14 years in boys and 11-12 years in girls)

3. Sex steroid priming for all prepubertal children (boys $>9$ years, girls $>8$ years, this can be based either on chronological age or bone age)

Table 1 Cut-off limits for the diagnosis of growth hormone deficiency for six commercially available growth hormone assays and one mass spectrometry assay

\begin{tabular}{ll}
\hline Assay & Cut-off limit $(\boldsymbol{\mu} \mathbf{g} / \mathbf{L})$ \\
\hline Immulite 2000 (Siemens) & 7.77 \\
AutoDELFIA (Perkin-Elmer) & 7.44 \\
Mass Spectrometry T12 fragment & 7.43 \\
iSYS (IDS) & 7.09 \\
Liasion (DiaSorin) & 6.25 \\
Mass Spectrometry T6 fragment & 5.48 \\
DxI (Beckman Coulter) & 5.15 \\
ELISA (Mediagnost) & 5.14 \\
BC-IRMA (Beckman Coulter) & 4.32 \\
\hline Data taken from Wagner et al. ${ }^{4}$ &
\end{tabular}

Internationally around a third to half of paediatric endocrinologists routinely prime peripubertal children prior to $\mathrm{GH}$ stimulation testing.

Common protocols for priming include intramuscular injection of testosterone (100 mg intramuscular, 7-10 days) before testing for boys and the administration of oral oestrogen (eg, 10-20 $\mu \mathrm{g}$ ethinyloestradiol) for $48-72 \mathrm{~h}$ prior to testing in girls. In order to avoid the need for an injection (potentially with an additional hospital visit) some paediatric endocrinologists will prime boys with oestrogen preparations such as stilboestrol or ethinyloestradiol.

\section{Obesity}

UK prevalence data indicate that $14 \%$ of UK children aged 215 years are classified as obese and $28 \%$ as overweight. Extensive data in the adult population indicated that spontaneous and stimulated $\mathrm{GH}$ secretion is reduced in obesity and has led to the development of different cut-off levels for the diagnosis of GHD in obese or overweight adults. ${ }^{19}$ Likewise it is clear in childhood that obesity is linked to reduced spontaneous $\mathrm{GH}$ secretion, ${ }^{20}$ reduced peak $\mathrm{GH}$ concentrations to stimulation testing $^{21}$ and increased rates of diagnosis of GHD compared with lean subjects of similar stature and IGF-I concentrations. ${ }^{21}$ A proportion of this increase in GHD diagnosis in obese subjects is likely to be the result of false positive tests due to their obesity, and there is therefore a need for BMI specific cut-off levels for peak $\mathrm{GH}$ concentrations during pharmacological stimulation testing in childhood and puberty.

\section{Measurement of IGF-I and IGFBP-3}

Serum IGF-I is mainly derived from the liver under the control of $\mathrm{GH}$ and circulates bound to the IGF binding proteins (IGFBPs). There are six classical IGFBPs of which IGFBP-3 is the major serum carrier of IGF-I. Unlike GH, serum concentrations of IGF-I and IGFBP-3 are stable throughout the day. Typically, total IGF-I concentrations in serum are measured via techniques that dissociate IGF-I from its binding proteins. The efficiency of this process or diseases affecting serum IGFBP concentrations, such as diabetes or renal impairment, can influence the total serum IGF-I concentration measured.

Serum IGF-I concentrations vary with age and, unfortunately, the normal range for serum IGF-I concentrations in young children overlaps with the range found in children with GHD. Additionally IGF-I concentrations are reduced in children with poor nutrition, hypothyroidism, chronic disease, renal failure and diabetes. They also rise dramatically during puberty; thus in the child with delayed puberty and low growth velocity the IGF-I concentration for age may appear low, although the bone age-adjusted and puberty stage-adjusted IGF-I concentration would be normal.

IGFBP-3 concentrations were thought to be potentially superior to measurement of IGF-I alone as IGFBP-3 is less nutritionally sensitive than IGF-I. Multiple studies have, however, found no difference in IGFBP-3 concentrations between GHD and non-GHD subjects, ${ }^{22}$ with a poor sensitivity at $50 \%$ and no advantage over measurement of IGF-I alone. ${ }^{23}$

\section{Neuroimaging}

The presence of an abnormality within the hypothalamopituitary axis provides powerful supporting evidence for a diagnosis of GHD. The most common radiological finding in GHD children is a variable combination of an ectopic posterior pituitary gland, anterior pituitary hypoplasia and a thin or interrupted pituitary stalk. ${ }^{24}$ Other abnormalities associated with 
GHD include hypothalamo-pituitary axis tumours such as craniopharyngioma, septo-optic dysplasia, corpus callosum hypoplasia/agenesis, holoprosencephaly, thickened pituitary stalk (seen in Langerhans cell histiocytosis and germinoma) and the presence of an empty sella.

\section{Genetic investigations}

Mutations in GH1, GHRHR and RNPC3 have been identified in patients with isolated $\mathrm{GHD}^{25}$ which may be associated with a normal MRI scan. ${ }^{25}$ The identification of a genetic mutation is particularly useful in supporting the diagnosis in cases of isolated GHD with a normal pituitary MRI. There are many other genes associated with GHD along with other pituitary deficiencies (POU1F1, PROP1, LHX3, LHX4, HESX1, OTX2, SOX2, SOX3, GLI2, GLI3, FGFR1, FGF8 and PROKR2) which are associated with additional clinical and radiological features. With the increasing clinical availability of genetic technologies such as whole exome and whole genome sequencing, screening for mutations to provide confirmation of the diagnosis of GHD is likely to increase.

\section{STARTING DOSE OF GH AND DOSE ADJUSTMENT}

Even within subjects with GHD there is a wide response to GH therapy, which is likely to be due to compliance issues, variability in the degree of GHD and in the patient's tissue responsiveness to GH. The licensed dose of GH for GHD is $0.7-1.0 \mathrm{mg} /$ $\mathrm{m}^{2} /$ day $(23-39 \mu \mathrm{g} / \mathrm{kg} /$ day $) .{ }^{1}$ While some endocrinologists will use a fixed dose of $\mathrm{GH}$, most will now use an auxology based dosing approach. This would normally involve starting at the lower end of this dose range and then titrating upwards (keeping within the dose range) based on the patient's response to therapy with measurement of IGF-I concentrations to ensure the patient was not overtreated or undertreated.

Dose titration was initially based on weight and growth velocity, but titration based on IGF-I concentrations has also been analysed. Where IGF-I titration is used, the clinician adjusts GH dose to a target IGF-I concentration irrespective of growth rate and usual range of GH dose. One open label IGF-I dosing trial randomising children to either a fixed dose or to targeted IGF-I concentrations of either $0 \mathrm{SD}$ or $+2 \mathrm{SD}^{26}$ found no significant differences in mean $\mathrm{GH}$ dose or 1 st year $\Delta$ Height SDS between the fixed dose and IGF-I targeted to 0 SD groups. The children targeted to an IGF-I concentration of +2 SD had a significantly improved 1st year $\Delta$ Height SDS, but required a mean GH dose of $91 \mu \mathrm{g} / \mathrm{kg} /$ day to achieve this. There are very few safety data using $\mathrm{GH}$ at such high doses and thus this approach cannot be recommended at the present time.

The major current alternative strategy to auxology or IGF-I based dosing is prediction model based dosing. There are three major prediction models for response to GH in GHD children (Kabi International Growth Study, Cologne and Göteburg models-the latter two models including biochemical tests not routinely performed in the UK) and each uses auxological and biochemical data. A prospective randomised trial comparing individualised dosing using the Göteburg prediction model to fixed weight based dosing did not identify any difference in overall growth response or GH dose between groups although the variability in response (the number of high and low responders) was reduced. ${ }^{27}$

\section{END OF GROWTH ASSESSMENT}

Currently, re-testing of $\mathrm{GH}$ status at end of growth is recommended by the European Society for Paediatric Endocrinology guidelines for all patients with GHD except those with panhypopituitarism (defined as four or five hormone deficiencies). ${ }^{28}$ Diagnosis of GHD during the transition period is beset by the same problems as those affecting the diagnosis of childhood GHD. Guidelines differ on suggested cut-offs for GHD diagnosis during transition, with American guidelines using adult cut-off values for the diagnosis of GHD $(3 \mu \mathrm{g} / \mathrm{L})$, European Society for Paediatric Endocrinology guidelines suggesting a cut-off of $5 \mu \mathrm{g} / \mathrm{L}$ and $\mathrm{GH}$ Research Society guidelines suggesting a cut-off of $6 \mu \mathrm{g} / \mathrm{L}$. The proportion of patients diagnosed as GHD who subsequently re-test with a peak GH $>10 \mu \mathrm{g} / \mathrm{L}$ (ie, not GHD by any cut-off) has been reported as high as $60 \%$ in patients with idiopathic isolated GHD and $22 \%$ among patients with an ectopic posterior pituitary. ${ }^{29}$ The reasons for the high proportion of patients who are apparently increasing their $\mathrm{GH}$ secretion are likely to be multifactorial. Long-term exposure to sex steroids may have augmented pituitary size and GH secretion, some children may have had a form of transient GHD and some patients may have been incorrectly diagnosed with GHD given the problems associated with biochemical testing outlined previously.

\section{POOR RESPONDERS TO GH TREATMENT}

Given the expense of $\mathrm{GH}$ treatment and the difficulties outlined with diagnostic tests, it is vital to identify those patients who respond poorly to treatment and reassess the diagnosis in this group. There are many suggested definitions of poor response to treatment (for review see Bang et $a l^{30}$ ) but no consensus on which definition to use. In the 2010 National Institute for Health and Care Excellence guidance, a recommendation was made to stop treatment where growth velocity increases less than $50 \%$ from baseline in the 1 st year of treatment. ${ }^{1}$ Depending on the definition used, the proportion of children with isolated GHD classified as poor responders can vary between $13 \%$ and $36 \% .{ }^{30}$ Variability in response to treatment may be due to poor compliance, environmental exposures or genetic differences in $\mathrm{GH}$ responsiveness.

\section{CONCLUSIONS AND RECOMMENDATIONS}

GHD remains a diagnosis principally based around the history, clinical features and auxology supported by biochemical and neuroradiological studies. Problems continue to exist with $\mathrm{GH}$ and IGF-I assays for standardisation, reproducibility and interassay variability. There are also problems with variability and reproducibility of the pharmacological stimulation tests. The best recent data on the selection of an optimal peak GH concentration comes from Wagner $e t a l^{4}$ and the cut-offs for each assay derived in that paper can be used where local assay and testspecific data are unavailable. With respect to priming, it is the authors' practice to prime with sex steroids prior to $\mathrm{GH}$ testing in all prepubertal girls aged $>8$ years and boys aged $>9$ years based on chronological age (box 2).

Most paediatric endocrinologists will select a starting dose at the lower end of the recommended range and titrate upwards based on auxology and IGF-I concentrations. Titrating to IGF-I concentrations appears to be no more effective than standard dosing unless the target is placed at the upper end of the IGF-I reference range. Such a target inevitably results in more children with supraphysiological IGF-I concentrations and the use of higher doses of $\mathrm{GH}$ where we have no long-term safety data. While prediction models can reduce the variability of response to $\mathrm{GH}$ therapy, they have not been shown to improve the overall response to treatment and also result in a small number of children being treated with doses of $\mathrm{GH}$ above those for which longterm safety data are available. The authors do not routinely use 


\section{Box 2 Authors' recommendations for clinical practice}

- Clinicians should be familiar with the details of the assays used in their local laboratories for growth hormone (GH), IGF-I and IGFBP-3.

- Where local assay and test specific data on cut-offs for stimulation tests are not available we would recommend the use of cut-off values described by Wagner et $\mathrm{al}^{4}$ (see table 1). Normative data are available for most IGF-I and IGFBP-3 assays. These should be used when interpreting results.

- The diagnosis of growth hormone deficiency (GHD) remains multifactorial and our practice is based around consensus guidelines $^{2}$ (see box 1). MRI should include specific views of the hypothalamo-pituitary axis.

- Our clinical practice is to prime all prepubertal patients (aged $>8$ years for girls or $>9$ years for boys) prior to $\mathrm{GH}$ stimulation testing.

- Our prescribing practice for GH therapy is based on weight or body surface area rather than IGF-I or prediction model based dosing (the latter is currently not easy to calculate in a clinic setting).

- At the initiation of GH therapy, there should be a discussion with the family/carers about expected outcomes and re-evaluation of the treatment decision based on response.

- The response to GH therapy should be assessed after 1 year of treatment and if unsatisfactory (eg, $<-2$ SD on published response curves ${ }^{30}$ ), adherence should be closely scrutinised and the diagnosis of GHD should be reconsidered.

prediction models to decide the starting dose of GH in their clinical practice (see Box 2 for authors' recommendations).

Identification of poor responders to GH therapy is an important area of clinical practice. Although there remains no consensus on definition of poor response, the possibility of such a response and the need to stop GH therapy and reassess diagnosis should be discussed with the parents/young person at the time of starting GH therapy. Where a GH dose is started at the lower end of the recommended range, at least 1 year of therapy will be required to titrate the dose upwards and to assess response on a higher dose. We would recommend that all children started on GH for GHD are seen at least every 4 months during the 1st year of therapy for this purpose.

Competing interests None declared.

Provenance and peer review Commissioned; externally peer reviewed.

\section{REFERENCES}

1 Human growth hormone (somatropin) for the treatment of growth failure in children. NICE, 2010. http://www.nice.org.uk/guidance/TA188

2 Growth Hormone Research $\mathrm{S}$. Consensus guidelines for the diagnosis and treatment of growth hormone (GH) deficiency in childhood and adolescence: summary statement of the GH Research Society. GH Research Society. J Clin Endocrinol Metab 2000;85:3990-3.

3 Kaplan SL, Abrams CA, Bell JJ, et al. Growth and growth hormone. I. Changes in serum level of growth hormone following hypoglycemia in 134 children with growth retardation. Pediatr Res 1968;2:43-63.

4 Wagner IV, Paetzold C, Gausche R, et al. Clinical evidence-based cutoff limits for $\mathrm{GH}$ stimulation tests in children with a backup of results with reference to mass spectrometry. Eur J Endocrinol 2014;171:389-97.
5 Shah A, Stanhope R, Matthew D. Hazards of pharmacological tests of growth hormone secretion in childhood. BMJ 1992;304:173-4.

6 Ghigo E, Bellone J, Aimaretti G, et al. Reliability of provocative tests to assess growth hormone secretory status. Study in 472 normally growing children. J Clin Endocrinol Metab 1996;81:3323-7.

7 Secco A, di lorgi N, Napoli F, et al. The glucagon test in the diagnosis of growth hormone deficiency in children with short stature younger than 6 years. J Clin Endocrinol Metab 2009:94:4251-7.

8 Hilczer M, Smyczynska J, Stawerska R, et al. Stability of IGF-I concentration despite divergent results of repeated GH stimulating tests indicates poor reproducibility of test results. Endocr Regul 2006;40:37-45.

9 Rose SR, Ross JL, Uriarte M, et al. The advantage of measuring stimulated as compared with spontaneous growth hormone levels in the diagnosis of growth hormone deficiency. N Engl J Med 1988;319:201-7.

10 Seth J, Ellis A, Al-Sadie R. Serum growth hormone measurements in clinical practice: an audit of performance from the UK National External Quality Assessment scheme. Horm Res 1999;51(Suppl 1):13-9.

11 Tanaka T, Tachibana K, Shimatsu A, et al. A nationwide attempt to standardize growth hormone assays. Horm Res 2005;64(Suppl 2):6-11.

12 Baumann G. Growth hormone heterogeneity: genes, isohormones, variants, and binding proteins. Endocr Rev 1991;12:424-49.

13 Ebdrup L, Fisker S, Sorensen $\mathrm{HH}$, et al. Variety in growth hormone determinations due to use of different immunoassays and to the interference of growth hormone-binding protein. Horm Res 1999;51(Suppl 1):20-6.

14 Rakover Y, Lavi I, Masalah R, et al. Comparison between four immunoassays for growth hormone $(\mathrm{GH})$ measurement as guides to clinical decisions following $\mathrm{GH}$ provocative tests. J Pediatr Endocrinol Metab 2000;13:637-43.

15 Arsene CG, Kratzsch J, Henrion A. Mass spectrometry -an alternative in growth hormone measurement. Bioanalysis 2014;6:2391-402.

16 Clayton PE, Price DA, Shalet SM. Growth hormone state after completion of treatment with growth hormone. Arch Dis Child 1987;62:222-6.

17 Marin G, Domene HM, Barnes KM, et al. The effects of estrogen priming and puberty on the growth hormone response to standardized treadmill exercise and arginine-insulin in normal girls and boys. J Clin Endocrinol Metab 1994;79:537-41.

18 Gonc EN, Kandemir N, Ozon A, et al. Final heights of boys with normal growth hormone responses to provocative tests following priming. J Pediatr Endocrinol Metab 2008;21:963-71.

19 Dichtel LE, Yuen KC, Bredella MA, et al. Overweight/obese adults with pituitary disorders require lower peak growth hormone cutoff values on glucagon stimulation testing to avoid overdiagnosis of growth hormone deficiency. J Clin Endocrinol Metab 2014:jc20142830

20 Martha PM Jr, Gorman KM, Blizzard RM, et al. Endogenous growth hormone secretion and clearance rates in normal boys, as determined by deconvolution analysis: relationship to age, pubertal status, and body mass. J Clin Endocrinol Metab 1992;74:336-44.

21 Stanley TL, Levitsky LL, Grinspoon SK, et al. Effect of body mass index on peak growth hormone response to provocative testing in children with short stature. J Clin Endocrinol Metab 2009:94:4875-81.

22 Phillip M, Chalew SA, Kowarski AA, et al. Plasma IGFBP-3 and its relationship with quantitative growth hormone secretion in short children. Clin Endocrinol 1993;39:427-32

23 Nunez SB, Municchi G, Barnes KM, et al. Insulin-like growth factor I (IGF-I) and IGF-binding protein-3 concentrations compared to stimulated and night growth hormone in the evaluation of short children-a clinical research center study. I Clin Endocrinol Metab 1996:81:1927-32.

24 Triulzi F, Scotti G, di Natale B, et al. Evidence of a congenital midline brain anomaly in pituitary dwarfs: a magnetic resonance imaging study in 101 patients. Pediatrics 1994;93:409-16.

25 Alatzoglou KS, Dattani MT. Genetic causes and treatment of isolated growth hormone deficiency-an update. Nat Rev Endocrinol 2010;6:562-76.

26 Cohen P, Germak J, Rogol AD, et al. Variable degree of growth hormone (GH) and insulin-like growth factor (IGF) sensitivity in children with idiopathic short stature compared with GH-deficient patients: evidence from an IGF-based dosing study of short children. J Clin Endocrinol Metab 2010;95:2089-98.

27 Kristrom B, Aronson AS, Dahlgren J, et al. Growth hormone (GH) dosing during catch-up growth guided by individual responsiveness decreases growth response variability in prepubertal children with GH deficiency or idiopathic short stature. J Clin Endocrinol Metab 2009:94:483-90.

28 Clayton PE, Cuneo RC, Juul A, et al. Consensus statement on the management of the GH-treated adolescent in the transition to adult care. Eur I Endocrinol 2005;152:165-70.

29 Leger J, Danner S, Simon D, et al. Do all patients with childhood-onset growth hormone deficiency (GHD) and ectopic neurohypophysis have persistent GHD in adulthood? I Clin Endocrinol Metab 2005;90:650-6.

30 Bang P, Bjerknes R, Dahlgren J, et al. A comparison of different definitions of growth response in short prepubertal children treated with growth hormone. Horm Res Paediatr 2011;75:335-45. 